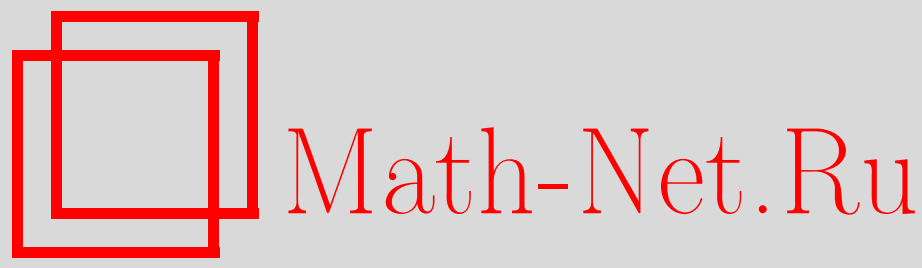

Л. Ц. Аджемян, М. Гнатич, М. В. Компаниец, Принцип максимальной хаотичности и нарушение четности в турбулентности, ТМФ, 2013, том 176, номер 1, 3-12

DOI: https://doi.org/10.4213/tmf8469

Использование Общероссийского математического портала Math-Net.Ru подразумевает, что вы прочитали и согласны с пользовательским соглашением http://www . mathnet.ru/rus/agreement

Параметры загрузки:

IP: 54.198 .187 .58

26 апреля 2023 г., 13:52:19

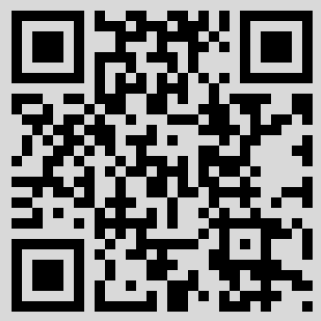




\title{
ПРИНЦИП МАКСИМАЛЬНОЙ ХАОТИЧНОСТИ И НАРУШЕНИЕ ЧЕТНОСТИ В ТУРБУЛЕНТНОСТИ
}

\begin{abstract}
Показано, что в уравнениях согласования для одновременны́х корреляционных функций флуктуаций скорости, полученных в модели развитой турбулентности, основанной на принципе максимальной хаотичности, инфракрасные расходимости отсутствуют во всех порядках теории возмущений. Проведен анализ дополнительных ультрафиолетовых расходимостей, возникающих в двухпетлевом приближении уравнений согласования. Показано, что в системе с ненарушенной четностью эти расходимости могут быть устранены имеющимся произволом в решении. Для систем с нарушенной четностью ультрафиолетовые расходимости носят логарифмический характер, что может указывать на отклонение от колмогоровского скейлинга.
\end{abstract}

Ключевые слова: уравнения согласования, колмогоровские степенные законы, спиральность, нарушение четности, инфракрасные и ультрафиолетовые расходимости.

DOI: $10.4213 / \operatorname{tmf} 8469$

\section{1. ВВЕДЕНИЕ}

При построении статистической теории развитой однородной изотропной турбулентности несжимаемой жидкости важную роль играют законы сохранения, определяющие параметры сокращенного описания системы. Ярким примером является феноменологическая теория Колмогорова. Эта теория основана на гипотезе о каскадном механизме переноса энергии по спектру турбулентных пульсаций скорости $\varphi$ от области наиболее крупных вихрей с характерным размером $L$ (область накачкu) до наиболее мелких, эффективно диссипирующих вихрей с характерным размером $l_{\text {diss }}$ (область диссипации). В развитой турбулентности с большими числами Рейнольдса $\operatorname{Re} \gg 1$ эти области сильно разнесены, $L / l_{\text {diss }} \sim \operatorname{Re}^{3 / 4} \gg 1$, так что существует достаточно большой интервал вихрей промежуточного размера - так

\footnotetext{
* Санкт-Петербургский государственный университет, Санкт-Петербург, Россия.
}

E-mail: loran.adzhemyan@gmail.com

${ }^{\dagger}$ Institute of Experimental Physics, Slovak Academy of Science, Košice, Slovakia. E-mail: hnatic@saske.sk

${ }^{\ddagger}$ Faculty of Sciences, P. J. Šafarik University, Košice, Slovakia. E-mail: michal.hnatic@upjs.sk 
называемый инерционный интервал. Главное предположение теории Колмогорова состоит в том, что в инерционном интервале и диссипативной области система после многочисленных актов дробления вихрей "забывает" детали области накачки и ее статистическое описание помимо внутреннего параметра - кинематической вязкости $\nu$ - определяется только интенсивностью потока энергии $W$ вдоль спектра размеров, равного скорости диссипации энергии на единицу массы $\bar{\varepsilon}$. Предсказания теории Колмогорова хорошо согласуются с экспериментальными данными, в частности подтверждается справедливость знаменитого “закона $5 / 3$ ” в инерционном интервале [1].

Кроме закона сохранения кинетической энергии для невязкой жидкости имеются специфические законы сохранения: в трехмерных течениях сохраняется так называемая спиральность $\chi=(\varphi, \operatorname{rot} \varphi)$, в двумерных течениях ${ }^{1)}$ - любые степени завихренности $\left(\operatorname{rot}^{2} \varphi\right)^{n}$. Среди последних наиболее важной величиной является энстрофия $\operatorname{rot}^{2} \boldsymbol{\varphi}$. Установлено, что наличие одновременно двух квадратичных по скорости интегралов движения приводит к тому, что для двумерных течений колмогоровская феноменология становится несправедливой, возникает инверсный поток энергии.

Более неопределенной является ситуация с возможным влиянием на статистические свойства турбулентности спиральности. Для систем с ненарушенной четностью среднее значение этой величины равно нулю, $\langle\boldsymbol{\varphi}, \operatorname{rot} \varphi\rangle=0$. Высказывались естественные предположения, что система может быть неустойчива по отношению к спонтанному нарушению четности и это среднее становится отличным от нуля. Возможное нарушение четности не сказывается на спектре энергии пульсаций, поэтому для проверки этой гипотезы требуются более детальные эксперименты. В настоящее время этот вопрос с точки зрения эксперимента остается открытым [2], [3]. Тем не менее экспериментально наблюдаемая значительная чувствительность свойств турбулентных течений к искусственному вращению системы (насильственному нарушению четности) косвенно подтверждает существенное влияние спиральности [4]-[6].

В работе [7] была предпринята попытка прямолинейной реализации идей Колмогорова о каскадном переносе энергии путем построения функции распределения одновременны́х пульсаций скорости на основе принципа максимальной хаотичности. Рассмотрение проводилось с использованием уравнений согласования. В теории турбулентности такой подход сталкивается с проблемой очень сильных инфракрасных (ИК) расходимостей. В работе [7] было показано, что в уравнениях, полученных в однопетлевом приближении, отсутствуют как ИК-расходимости, так и ультрафиолетовые (УФ) расходимости. В инерционном интервале эти уравнения имели степенные решения с колмогоровскими показателями. Однако полученное решение не согласовывалось с положительной определенностью спектра энергии, в связи с чем авторы предприняли попытку найти решение с нарушенной четностью. Такое решение было получено. Оно также оказалось свободным от расходимостей, но коэффициент, описывающий величину нарушения четности, на $3 \%$ превышал максимально допустимое значение. Такое превышение могло быть следствием приближенного характера рассмотрения (однопетлевое приближение). Его малое значение позволяло надеяться, что дефект может быть исправлен учетом двухпетлевых поправок.

1) Более точно, в системах, в которых пульсации скорости $\varphi$ лежат в плоскости и не зависят от поперечной координаты. 
Переход к двухпетлевому приближению, помимо чисто технических трудностей расчета диаграмм, требует решения вопроса об отсутствии ИК-расходимостей и дополнительных УФ-расходимостей. В настоящей работе на основе рассмотрения общих свойств вершины взаимодействия будет доказано отсутствие ИК-расходимостей во всех порядках теории возмущений и проанализированы УФ-расходимости, которые появляются в уравнениях согласования в двухпетлевом приближении.

\section{2. МОДЕЛЬ МАКСИМАЛЬНОЙ ХАОТИЧНОСТИ}

Уравнение Навье-Стокса для случайного поля скоростей несжимаемой жидкости эквивалентно бесконечной системе уравнений для корреляционных функций. Первое уравнение для рассматриваемого стационарного случая $\partial_{t}\left\langle E_{i j}(\mathbf{k})\right\rangle=0$ имеет вид

$$
\left\langle\dot{E}_{i j}(\mathbf{k})\right\rangle=-d_{i j}(\mathbf{k}), \quad \dot{E}_{i j}(\mathbf{k})=-\nu k^{2} E_{i j}(\mathbf{k})-T_{i j}(\mathbf{k}),
$$

где $\nu$ - кинематическая вязкость,

$$
E_{i j}(\mathbf{k})=\varphi_{i}(\mathbf{k}) \varphi_{j}(-\mathbf{k}), \quad T_{i j}(\mathbf{k})=\frac{1}{2}\left[\varphi_{i}(\mathbf{k})\left(\varphi_{s} \partial_{s} \varphi_{j}\right)(-\mathbf{k})+\varphi_{j}(-\mathbf{k})\left(\varphi_{s} \partial_{s} \varphi_{i}\right)(\mathbf{k})\right] .
$$

Величина $d_{i j}(\mathbf{k})$ в уравнении (1) моделирует внешнюю накачку энергии из длинноволновой области. Если исследовать инерционный и диссипативный интервал, эту величину можно представить в виде

$$
d_{i j}(\mathbf{k})=\frac{P_{i j}(2 \pi)^{d} W \delta(\mathbf{k})}{d-1}
$$

где $d$-размерность пространства волновых векторов.

Искомая функция распределения $\varrho(\varphi)$ строится на основе требования максимума информационной энтропии

$$
\sigma=-\langle\ln \varrho\rangle=-\int D \varphi \varrho(\varphi) \ln \varrho(\varphi)
$$

и одновременного выполнения уравнения (1). Вводя множители Лагранжа $\lambda_{i j}(\mathbf{k})$ и переходя к задаче на безусловный экстремум, получаем

$$
\varrho(\varphi)=Q^{-1} \exp \left[(2 \pi)^{-d} \int d(\mathbf{k}) \lambda_{i j}(\mathbf{k}) \dot{E}_{i j}(\mathbf{k})\right],
$$

где $Q$ - нормировочный множитель. Множители $\lambda_{i j}(\mathbf{k})$ должны быть определены из уравнения (1). Так как $\dot{E}_{i j}(\mathbf{k})=\dot{E}_{j i}(-\mathbf{k})$, можно положить

$$
\lambda_{i j}(\mathbf{k})=\lambda_{j i}(-\mathbf{k})
$$

и получить квантово-полевую модель

$$
\varrho=Q^{-1} e^{S}
$$

с действием

$$
S=-\int \frac{d \mathbf{k}}{(2 \pi)^{d}}\left[\nu k^{2} \lambda_{i j}(\mathbf{k}) \varphi_{j}(\mathbf{k}) \varphi_{i}(-\mathbf{k})+\lambda_{i j}(\mathbf{k}) \varphi_{j}(\mathbf{k})\left(\varphi_{s} \partial_{s} \varphi_{i}\right)(-\mathbf{k})\right] .
$$


Уравнение спектрального баланса энергии получается из уравнения (1) сворачиванием векторных значков. Величина $\left\langle E_{i i}(\mathbf{k})\right\rangle$ определяет тогда спектральную плотность энергии $E(k)$, а величина $\left\langle T_{i i}(\mathbf{k})\right\rangle=T(k)$ - функцию переноса. В однородной изотропной системе с ненарушенной четностью все величины в уравнении (1) пропорциональны поперечному проектору $P_{i j}(\mathbf{k})=\delta_{i j}-k_{i} k_{j} / k^{2}$, что является следствием условия несжимаемости для поля $\boldsymbol{\varphi}$, поэтому запись (1) эквивалентна соотношению (1) со свернутыми векторными значками. Мы используем запись (1) с целью сохранить возможность рассмотреть в дальнейшем также ситуацию с нарушенной четностью. В этом случае все члены в уравнении (1) содержат также вклад $\sim i \varepsilon_{i j l} k_{l}$. Соответствующая скалярная величина $i \varepsilon_{i j l} k_{l}\left\langle E_{i j}(\mathbf{k})\right\rangle$ определяет спектральную плотность спиральности $\chi=(\boldsymbol{\varphi}, \operatorname{rot} \boldsymbol{\varphi})$, а величина $i \varepsilon_{i j l} k_{l}\left\langle T_{i j}(\mathbf{k})\right\rangle \equiv T_{\chi}(k)-$ соответствующую функцию переноса.

\section{3. УРАВНЕНИЯ СОГЛАСОВАНИЯ}

Для получения уравнений согласования перепишем действие (7) в более удобной форме с локальным взаимодействием. Для этого при вычислениях стандартным образом вводится единичная операция $\prod D u_{i} \delta\left(u_{i}(\mathbf{k})-\lambda_{i j}(\mathbf{k}) \varphi(\mathbf{k})\right)$. Дельта-функцию $\delta(u-\lambda \varphi)$ можно записать в фурье-представлении с помощью вспомогательного поля $\mathbf{w}$ (и затем $i \mathbf{w} \rightarrow \mathbf{w}$ ). Это позволяет ввести функцию распределения трех поперечных полей $\boldsymbol{\varphi}, \mathbf{u}$ и $\mathbf{w}$ с помощью соотношения $\rho(\boldsymbol{\varphi}, \mathbf{u}, \mathbf{w})=Q^{-1} e^{S}$, где

$$
S=-\nu\left(\partial_{j} u_{i}\right)\left(\partial_{j} \varphi_{i}\right)+\left(\partial_{j} u_{i}\right) \varphi_{i} \varphi_{j}-w_{i} \lambda_{i j} \varphi_{j}+w_{i} u_{i}
$$

На языке модели (8) условие (1) приобретает вид [7]

$$
\left\langle\varphi_{i}(\mathbf{k}) w_{j}(-\mathbf{k})\right\rangle=d_{i j}(\mathbf{k})
$$

Обратная матрица корреляторов полей $\boldsymbol{\varphi}, \mathbf{u}, \mathbf{w}$ в импульсном представлении имеет вид

$$
\widehat{G}^{-1}=\left(\begin{array}{ccc}
-\Sigma^{\varphi \varphi} & \nu k^{2}-\Sigma^{\varphi u} & \lambda \\
\nu k^{2}-\Sigma^{u \varphi} & -\Sigma^{u u} & -1 \\
\lambda & -1 & 0
\end{array}\right) .
$$

Обращая матрицу (10), получаем

$$
\left(G^{\varphi \varphi}\right)^{-1}=-\Sigma^{\varphi \varphi}-\lambda^{2} \Sigma^{u u}+\lambda\left(2 \nu k^{2}-\Sigma^{u \varphi}-\Sigma^{\varphi u}\right) .
$$

Соотношение (9) с учетом (10), (11) приобретает вид

$$
G^{\varphi \varphi}\left(\nu k^{2}-\Sigma^{u \varphi}-\lambda \Sigma^{u u}\right)=d(k) .
$$

\section{4. ОТСУТСТВИЕ ИК-РАСХОДИМОСТЕЙ В МОДЕЛИ МАКСИМАЛЬНОЙ ХАОТИЧНОСТИ}

На основе соотношений (6), (7) мы получим уравнения согласования. В процессе поиска их решения появляются ИК- и УФ-расходимости. Для успешного преодоления этих проблем следующие три свойства вершины взаимодействия в действии (7) будут иметь решающее значение. 
1. Вершина такова, что она обращается в нуль, как только обращается в нуль импульс одной из скоростей, в нее входящих. Это означает, что в диаграммах с одетыми линиями сингулярность безмассовых линий не приводит к появлению ИК-расходимостей.

Записывая действие (7) в импульсном представлении, получаем

$$
S_{\mathrm{int}}=-i \int \frac{d \mathbf{k}}{(2 \pi)^{d}} \int \frac{d \mathbf{q}}{(2 \pi)^{d}}\left(q_{s}-k_{s}\right) \lambda_{i j}(\mathbf{k}) \varphi_{j}(\mathbf{k}) \varphi_{s}(-\mathbf{q}) \varphi_{i}(\mathbf{q}-\mathbf{k}) .
$$

Заменяя здесь $\mathbf{k}$ на $\mathbf{q}-\mathbf{k}$ (в то же время $\mathbf{q}-\mathbf{k}$ заменяется на $\mathbf{k}$ ) и переставляя местами индексы суммирования $i \leftrightarrow j$, получаем

$$
S_{\mathrm{int}}=-i \int \frac{d \mathbf{k}}{(2 \pi)^{d}} \int \frac{d \mathbf{q}}{(2 \pi)^{d}} k_{s} \lambda_{j i}(\mathbf{q}-\mathbf{k}) \varphi_{i}(\mathbf{q}-\mathbf{k}) \varphi_{s}(-\mathbf{q}) \varphi_{j}(\mathbf{k}) .
$$

Выражения (13), (14) равны, поэтому действие может быть записано как их полусумма. Принимая во внимание, что слагаемое в $(13)$, содержащее $q_{s}$, обращается в нуль в силу поперечности поля скорости $\left(q_{s} \varphi_{s}(-\mathbf{q})=0\right)$, приходим к действию

$$
S_{\mathrm{int}}=\frac{i}{2} \int \frac{d \mathbf{k}}{(2 \pi)^{d}} \int \frac{d \mathbf{q}}{(2 \pi)^{d}} k_{s} \varphi_{j}(\mathbf{k}) \varphi_{s}(-\mathbf{q}) \varphi_{i}(\mathbf{q}-\mathbf{k})\left[\lambda_{i j}(\mathbf{k})-\lambda_{j i}(\mathbf{q}-\mathbf{k})\right] .
$$

Используя свойство (5) множителей Лагранжа, перепишем последнее выражение в окончательном виде:

$$
S_{\mathrm{int}}=\frac{i}{2} \int \frac{d \mathbf{k}}{(2 \pi)^{d}} \int \frac{d \mathbf{q}}{(2 \pi)^{d}} k_{s} \varphi_{j}(\mathbf{k}) \varphi_{s}(-\mathbf{q}) \varphi_{i}(\mathbf{q}-\mathbf{k})\left[\lambda_{i j}(\mathbf{k})-\lambda_{i j}(\mathbf{k}-\mathbf{q})\right] .
$$

Из (15) видно, что вершина обращается в нуль, как только аргумент какой-либо скорости стремится к нулю. Для $\varphi_{j}(\mathbf{k})$ это следует из присутствия множителя $k_{s}$, для $\varphi_{s}(-\mathbf{q})-$ из обращения в нуль скобки $\left[\lambda_{i j}(\mathbf{k})-\lambda_{i j}(\mathbf{k}-\mathbf{q})\right]$ при $\mathbf{q}=0$, а для $\varphi_{i}(\mathbf{q}-\mathbf{k})$ - из условия ортогональности $k_{s} \varphi_{s}(-\mathbf{q})=0$ при $\mathbf{k}=\mathbf{q}$.

2. Второе свойство связано с законом сохранения энергии: слагаемое в $\lambda_{i j}(\mathbf{k})$ вида $P_{i j}(\mathbf{k}) \cdot$ const не дает вклада в (15). Действительно, в этом случае оба проектора в разности $P_{i j}(\mathbf{k})-P_{i j}(\mathbf{k}-\mathbf{q})$ можно заменить на $\delta_{i j}$ : первый из-за свертки в $(15)$ с поперечным полем $\varphi_{j}(\mathbf{k})$, а второй из-за свертки с поперечным полем $\varphi_{i}(\mathbf{q}-\mathbf{k})$. После этого разность проекторов обращается в нуль.

3. Третье свойство связано с законом сохранения спиральности: взаимодействие (15) обращается в нуль также для вклада $\varepsilon_{i j l} k_{l}$ в $\lambda_{i j}(\mathbf{k})$. Подставляя такой вклад в разность $\lambda_{i j}(\mathbf{k})-\lambda_{i j}(\mathbf{k}-\mathbf{q})$ и сокращая члены, пропорциональные импульсу $k$, получаем

$$
\int d \mathbf{k} \int d \mathbf{q} k_{s} \varphi_{j}(\mathbf{k}) \varphi_{s}(-\mathbf{q}) \varphi_{i}(\mathbf{q}-\mathbf{k}) \varepsilon_{i j l} q_{l} .
$$

Заменим $k_{s} \varphi_{j}(\mathbf{k})$ на $k_{s} \varphi_{j}(\mathbf{k})-k_{j} \varphi_{s}(\mathbf{k})$ в $(16)$. Это можно сделать "бесплатно", так как добавочное слагаемое дает нулевой вклад в $(16):$ замена $\mathbf{k} \rightarrow-\mathbf{q}, \mathbf{q} \rightarrow-\mathbf{k}, l \leftrightarrow j$ (в то же время $\mathbf{q}-\mathbf{k} \rightarrow \mathbf{q}-\mathbf{k}, k_{j} q_{l} \rightarrow k_{j} q_{l}$ ) в выражении

$$
\int d \mathbf{k} \int d \mathbf{q} k_{j} \varphi_{s}(\mathbf{k}) \varphi_{s}(-\mathbf{q}) \varphi_{i}(\mathbf{q}-\mathbf{k}) \varepsilon_{i j l} q_{l}
$$


показывает, что единственное изменение состоит в перестановке индексов $l \leftrightarrow j$ в $\varepsilon_{i j l}$, что меняет знак всего выражения, поэтому оно должно быть равно нулю. Запишем соотношение

$$
k_{s} \varphi_{j}(\mathbf{k})-k_{j} \varphi_{s}(\mathbf{k})=\left(\delta_{s m} \delta_{j n}-\delta_{s n} \delta_{j n}\right) k_{m} \varphi_{n}(\mathbf{k})=\varepsilon_{t s j} \varepsilon_{t m n} k_{m} \varphi_{n}(\mathbf{k})
$$

и подставим его в (16):

$$
\int d \mathbf{k} \int d \mathbf{q} \varepsilon_{t s j} \varepsilon_{t m n} k_{m} \varphi_{n}(\mathbf{k}) \varphi_{s}(-\mathbf{q}) \varphi_{i}(\mathbf{q}-\mathbf{k}) \varepsilon_{i j l} q_{l} .
$$

Используя свертку $\varepsilon_{t s j} \varepsilon_{i j l}=\delta_{s i} \delta_{t l}-\delta_{s l} \delta_{t i}$ и тот факт, что вклад, пропорциональный $\delta_{s l}$, обращается в нуль в силу $q_{s} \varphi_{s}(-\mathbf{q})=0$ в $(17)$, приходим к выражению

$$
\int d \mathbf{k} \int d \mathbf{q} \varepsilon_{l m n} k_{m} \varphi_{n}(\mathbf{k}) \varphi_{i}(-\mathbf{q}) \varphi_{i}(\mathbf{q}-\mathbf{k}) q_{l} .
$$

Произведя в (18) подстановку $-\mathbf{q}=\tilde{\mathbf{q}}-\mathbf{k}($ соответственно $\mathbf{q}-\mathbf{k}=-\tilde{\mathbf{q}})$ и принимая во внимание $\varepsilon_{l n m} k_{m} q_{l} \rightarrow \varepsilon_{l n m} k_{m}\left(k_{l}-\tilde{q}_{l}\right)=-\varepsilon_{l n m} k_{m} \tilde{q}_{l}$, убеждаемся, что выражение (18) сменило знак, и, следовательно, оно равно нулю.

\section{5. РЕШЕНИЕ В ИНЕРЦИОННОМ ИНТЕРВАЛЕ}

Если в выражениях для собственно энергетических вкладов $\Sigma$ мы ограничимся однопетлевыми скелетными диаграммами, то из (11), (12) получим уравнения согласования в однопетлевом приближении:

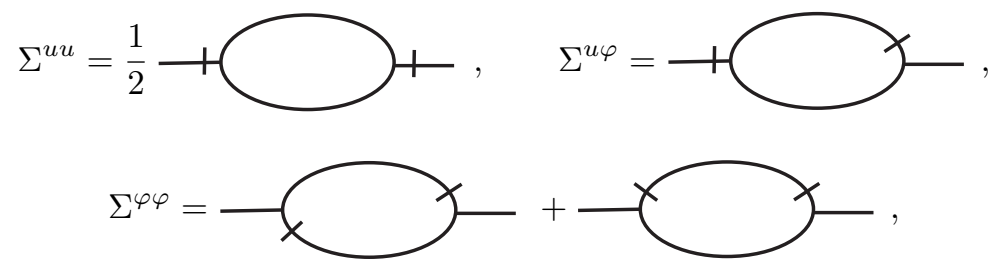

где введены графические обозначения для симметризованной вершины взаимодействия и полных пропагаторов:

$$
\begin{aligned}
& \stackrel{s}{+} \underbrace{i}_{j}=\frac{1}{2} \varphi_{i} \varphi_{j}\left(\partial_{i} \delta_{j s}+\partial_{j} \delta_{i s}\right) u_{s}, \\
& =G^{\varphi \varphi}, \quad=G^{\varphi u}, \quad=G^{u u} \text {. }
\end{aligned}
$$

В инерционном интервале $d_{i j}=0, \nu=0$. Это соответствует постоянству потока энергии по спектру. Чтобы фиксировать величину потока, введем в инерционном интервале бесконечно малый источник, представив $\delta$-функцию в (2) в виде

$$
\delta(\mathbf{k})=\frac{1}{S_{d}} \lim _{\varepsilon \rightarrow+0} \varepsilon k^{\varepsilon-d} .
$$

Для системы с ненарушенной четностью будем искать решение уравнений (11), (12) в виде

$$
G_{i j}(\mathbf{k})=b P_{i j}(\mathbf{k}) k^{-\gamma}, \quad \lambda_{i j}(\mathbf{k})=a P_{i j}(\mathbf{k}) k^{\beta}
$$


с подлежащими определению показателями $\gamma, \beta$ и амплитудами $a, b$. Подставляя (23) в (11), (12) и приравнивая показатели степеней, находим

$$
\gamma=d+\frac{2}{3}-\frac{2}{3} \varepsilon, \quad \beta=d-\varepsilon .
$$

При $\varepsilon=0$ для показателя $\gamma$ получаем колмогоровское значение $\gamma=d+2 / 3$. Уравнение (12) для случая ненарушенной четности эквивалентно равенству $T(k)=d(k)$. Мы получаем $T(k)=0$ для $\gamma=d+2 / 3$ благодаря свойству $\int d \mathbf{k} T(k)=0$. Это обеспечивает конечность амплитуд при предельном переходе $\varepsilon \rightarrow 0$ в уравнении $T(k)=d(k)$.

Сделанные оценки справедливы при условии ИК- и УФ-сходимости интегралов. При использовании уравнений согласования в теории турбулентности наиболее серьезные затруднения связаны с проблемой ИК-сходимости [8]-[10]. В модели максимальной хаотичности этой проблемы нет благодаря свойству 1 вершины взаимодействия (см. раздел 4). Однако степенной рост множителей Лагранжа $\lambda_{i j}(\mathbf{k}) \sim k^{d}$ приводит к УФ-расходимости некоторых диаграмм. Вводя регуляризацию $\Lambda$ обрезанием на больших импульсах, можно показать, что расходящаяся часть диаграмм с учетом свойства 1 вершины взаимодействия пропорциональна $k^{2} \Lambda^{5 / 3}$. Необходимый контрчлен, сокращающий эту расходимость, удается получить, включив дополнительное слагаемое в множитель Лагранжа:

$$
\lambda_{i j}(\mathbf{k})=A P_{i j}(\mathbf{k})+a P_{i j}(\mathbf{k}) k^{\beta} .
$$

Согласно свойству 2 слагаемое $A P_{i j}(\mathbf{k})$ не дает вклада в вершину взаимодействия. Оно дает вклад лишь в квадратичную часть действия. Именно эта часть является источником необходимых контрчленов, а пропагатор $G_{i j}(\mathbf{k})$ сохраняет прежний степенной вид с колмогоровским показателем.

Проведенный в работе [7] однопетлевой расчет показал, что амплитуда $b$ положительно-определенного коррелятора $G^{\varphi \varphi}$ в (23) принимает положительные значения лишь в интервале $2<d<d_{*}$, она обращается в нуль в точке $d_{*} \simeq 2.56$ и стремится к бесконечности при $d \rightarrow 2$. Последнее обстоятельство можно интерпретировать как влияние дополнительного закона сохранения энстрофии при $d=2$ : при $d \rightarrow 2$ происходит "запирание" потока энергии, т. е. он не может попасть в область больших волновых чисел, это приводит к росту амплитуды в инерционном интервале и к появлению при $d=2$ инверсного потока. Отсутствие физического решения при $d>d_{*}$ может быть связано с тем, что не учтено влияние дополнительного закона сохранения спиральности при $d=3$. В работе [7] авторы предположили, что это влияние проявляется в виде спонтанного нарушения четности, и искали решение в виде

$$
\begin{aligned}
G_{i j}(\mathbf{k}) & =b k^{-\gamma}\left(P_{i j}+i \theta \varepsilon_{i j s} \frac{k_{s}}{k}\right), \\
\lambda_{i j}(\mathbf{k}) & =A P_{i j}+i B \varepsilon_{i j s} k_{s}+a k^{\beta}\left(P_{i j}+i \varsigma \varepsilon_{i j s} \frac{k_{s}}{k}\right),
\end{aligned}
$$

где $\varepsilon_{i j l}$ - полностью антисимметричный тензор. Слагаемое $i B \varepsilon_{i j s} k_{s} / k$ включено в $\lambda_{i j}$ для получения необходимых контрчленов, устраняющих дополнительные УФрасходимости, в силу свойства 3 оно не дает вклада в вершину взаимодействия. 
Решению (26), (27) отвечают прежние значения показателей (24). Отметим, что при $d=3, \varepsilon=0$ из $(24)$ имеем $\beta=3$, и нарушающий четность вклад во взаимодействие определяется локальным выражением

$$
(\operatorname{rot} \varphi)_{j} \partial^{2} \partial_{s} \varphi_{j} \varphi_{s}
$$

Найденное в [7] в однопетлевом приближении решение вида (26), (27) содержит внутреннее противоречие: для параметра $\theta$, определяющего степень нарушения четности, было получено значение $\theta \simeq \pm 1.03$, несколько выходящее за пределы интервала разрешенных значений $|\theta|<1$, диктуемого требованием положительной определенности величины $G_{i j}(\mathbf{k})$. Ситуация здесь до некоторой степени аналогична той, которая имела место в работе [10], однако в [10] было получено значение $\theta=2.83$, далеко выходящее за разрешенные рамки, и это нельзя было трактовать как следствие приближенного характера уравнений (однопетлевое приближение). В рассматриваемом же случае учет двухпетлевых поправок вполне может исправить ситуацию, это делает проведение такого расчета актуальной задачей.

\section{6. ДВУХПЕТЛЕВОЕ ПРИБЛИЖЕНИЕ}

Двухпетлевое приближение уравнений согласования с одетыми линиями определяется диаграммами вида

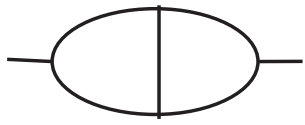

со всевозможными перечеркиваниями одной из линий в каждой вершине. Необходимо убедиться в ИК- и УФ-сходимости этих диаграмм на решении (26), (27) с показателями (24). Рассмотрим случай $d=3$. Отсутствие ИК-расходимостей в рассматриваемых диаграммах гарантируется свойством 1 вершины взаимодействия. Поверхностная УФ-расходимость носит тот же характер, что и в однопетлевом приближении и, следовательно, может быть устранена введением дополнительных вкладов в контрчленах в (27). В дополнительном анализе нуждаются УФ-расходимости, возникающие в треххвостых подграфах

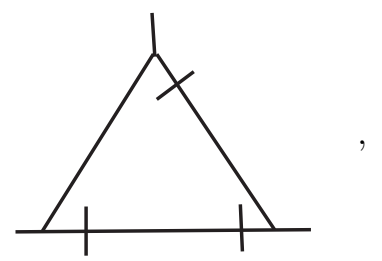

в которых, как в приведенном примере, все перечеркивания сосредоточены на внутренних линиях. Представим сумму таких диаграмм в виде

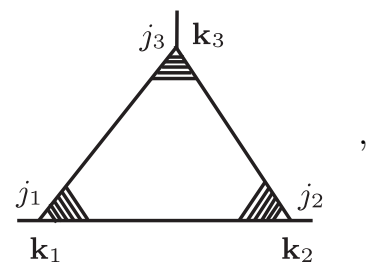


где $\mathbf{k}_{s}$ - импульс, втекающий в вершину $\left(\mathbf{k}_{1}+\mathbf{k}_{2}+\mathbf{k}_{3}=0\right), j_{s}$ - поляризация соответствующей внешней линии. Контрчлен к диаграмме представляет собой свертку расходящейся части диаграммы с тремя внешними полями и является полиномом по внешним импульсам, по меньшей мере третьего порядка в силу свойства 1 вершины взаимодействия. В координатном представлении этому удовлетворяет локальная конструкция вида $\partial \varphi \partial \varphi \partial \varphi$ со свернутыми значками, которая не сводится к полной производной. На первый взгляд, с учетом поперечности поля $\varphi$, возможны два варианта сворачивания значков: $\partial_{j_{1}} \varphi_{j_{2}} \partial_{j_{1}} \varphi_{j_{3}} \partial_{j_{3}} \varphi_{j_{2}}$ и $\partial_{j_{1}} \varphi_{j_{2}} \partial_{j_{2}} \varphi_{j_{3}} \partial_{j_{3}} \varphi_{j_{1}}$. Однако второй вариант сводится к полной производной, поэтому его можно не учитывать. Остающийся контрчлен с точностью до полной производной и знака сводится к величине $\varphi_{j_{2}} \partial^{2} \partial_{j_{3}} \varphi_{j_{3}} \varphi_{j_{2}}$. Такой контрчлен воспроизводится в вершине взаимодействия добавкой к $\lambda_{i j}(\mathbf{k})$ аналитического вклада $\sim k^{2} P_{i j}(\mathbf{k})$.

В случае нарушения четности контрчлен может содержать также вектор $\boldsymbol{\Omega}=$ $\operatorname{rot} \varphi$. Нетривиальным является только контрчлен вида $\partial \Omega \partial \varphi \partial \varphi-$ контрчлен $\partial \Omega \partial \Omega \partial \varphi$ сводится к $\partial \varphi \partial \varphi \partial \varphi$, а вариант $\partial \Omega \partial \Omega \partial \Omega$ соответствует сходящемуся интегралу. Контрчлен $\partial \Omega \partial \varphi \partial \varphi$ имеет ту же структуру, что и локальный вклад во взаимодействие (28), ему соответствует логарифмическая УФ-расходимость. Такая ситуация требует тщательного дополнительного рассмотрения. Она может свидетельствовать о сдвиге показателей с колмогоровского значения (как в аналогичном случае получения $1 / n$-разложения с помощью уравнений согласования [11]).

\section{7. ЗАКЛЮЧЕНИЕ}

Статистический ансамбль, использованный в настоящей работе, является простейшей реализацией идеи Колмогорова об определяющей роли спектрального баланса энергии в статистическом описании развитой турбулентности. Сочетание этой идеи с принципом максимальной хаотичности приводит к функции распределения поля скорости, сходной с функциями распределения Гиббса. Роль полной энергии и сопряженной с ней обратной абсолютной температуры играет спектральный поток энергии и соответствующий ему сопряженный параметр. Уравнения согласования в такой модели свободны от ИК-расходимостей.

Согласно результатам однопетлевого расчета только решение с нарушенной четностью является стабильным. В то же время двухпетлевые вклады, нарушающие четность, приводят к логарифмическим УФ-расходимостям, что может свидетельствовать о сдвиге показателей с колмогоровских значений. Мы намерены провести численные расчеты в двухпетлевом приближении в ближайшем будущем.

В заключение обсудим возможность появления ИК- и УФ-расходимостей в старших порядках уравнений согласования. На самом деле с ИК-расходимостями не возникает никаких проблем, так как ИК-сходимость диаграмм гарантирована свойством 1 вершины взаимодействия. С УФ-расходимостями ситуация более сложная. Их присутствие определяется одночастично-неприводимыми $n$-точечными функциями Грина вида (20) и (30). Их поверхностная УФ-расходимость определяется размерностью $\Delta_{n}$. Благодаря тому что на каждую внешнюю линию выделяется внешний импульс, эффективная размерность равна $\Delta_{n}^{\mathrm{eff}}=3-2 n / 3$. Для $n>4$ имеем $\Delta_{n}^{\mathrm{eff}}<0$, и поверхностная расходимость отсутствует. Таким образом, помимо рассмотренных случаев $n=2$ и $n=3$ опасными являются только четырехточечные 
функции с $\Delta_{4}^{\mathrm{eff}}=1 / 3$. Для контрчленов с нарушенной четностью, т. е. для тех, которые содержат вектор $\boldsymbol{\Omega}$, эффективная размерность уменьшается на единицу, следовательно, поверхностная расходимость также отсутствует. Мы можем заключить, что только одночастично-неприводимые четырехточечные функции с ненарушенной четностью должны быть проанализированы позже.

Благодарности. Работа поддержана РФФИ (грант № 12-02-00874_a), а также Ministry of Education, Science, Research and Sport of the Slovak Republic (VEGA грант 1/0222/13) и Centre of Excellency for Nanofluid of IEP SAS. Настоящая работа также финансировалась проектами № 26220120033, 26220120021 и 26110230061 по внедрению кооперативных явлений и фазовых переходов в наносистемах с перспективой использования в нано- и биотехнологиях. Программа исследования и развития финансировалась European Regional Development Fund.

\section{Список литературы}

[1] A.S. Monin, A. M. Yaglom, Statistical Fluid Mechanics: Mechanics of Turbulence, Dover, Mineola, NY, 2007.

[2] E. Kit, A. Tsinober, J. L. Balint, J. M. Wallace, E. Levich, Phys. Fluids, 30:11 (1987), 3323-3325.

[3] J. M. Wallace, J.-L. Balint, L. Ong, Phys. Fluids A, 4:9 (1992), 2013, 14 pp.

[4] M. Thiele, W.-C. Müller, J. Fluid Mech., 637 (2009), 425-442, arXiv: 0906.0853.

[5] C. Morize, F. Moisy, Phys. Fluids, 18:6 (2006), 065107, 9 pp.

[6] Y. Morinishi, K. Nakabayashi, S. Q. Ren, Phys. Fluids, 13:10 (2001), 2912-2922.

[7] L. Ts. Adzhemyan, M. Hnatich, M. Stehlik, J. Phys. II France, 5:7 (1995), 1077-1092.

[8] S. F. Edwards, W. D. McComb, J. Phys. A, 2:2 (1969), 157-171.

[9] A. Yoshizawa, J. Phys. Soc. Japan, 40:5 (1976), 1498-1503.

[10] A. A. Migdal, Modern Phys. Lett. A, 6:11 (1991), 1023-1043.

[11] А.Н. Васильев, Квантовополевая ренормгруппа в теории критического поведения и стохастической динамике, Изд-во ПИЯФ, СПб., 1998. 